Proc., ONR Solid Mechanics Program Annual Review Meeting,

Y.D.S. Rajapakse, ed., University of Maryland, pp. 12--20

\title{
SIZE EFFECT OF COHESIVE DELAMINATION FRACTURE TRIGGERED BY SANDWICH SKIN WRINKLING
}

\author{
Z. P. Bažant ${ }^{1}$ and P. Grassl ${ }^{2}$ \\ ${ }^{1}$ Northwestern University, CEE, 2145 Sheridan Road, Evanston, Illinois 60208, U.S.A. \\ ${ }^{2}$ Northwestern University, Evanston (currently University of Glasgow, Glasgow, U.K.)
}

OBJECTIVE: The objective of this paper is study the size effect on the nominal strength of imperfect sandwich structures for buckling driven delamination, to quantify this size effect, and to determine its intensity. A secondary objective is to assess the size effect on the postpeak energy absorption, important for judging survival under blast or dynamic impact.

\begin{abstract}
APPROACH: Buckling driven delamination is difficult to control in experiments. Thus, the present study relies on numerical simulations using geometrically nonlinear finite element analysis as well as the softening foundation model, which is an adaptation of Winkler elastic foundation. Delamination fracture is modeled by a cohesive crack model. Dimensionless variables are used to cover the entire practical range.
\end{abstract}

RECENT ACHIEVEMENTS:A major question in extrapolating small-scale laboratory tests to full-scale sandwich structures is the size effect. Delamination of the skin (or facesheet) is often triggered by wrinkling instability, which has generally been considered to be free of size effect. The absence of size effect has been inferred from the fact that the critical stress for buckling generally exhibits no size effect. However, this inference is valid only for the symmetry-breaking bifurcation of equilibrium path in perfect structures. Actual sandwich structures are always imperfect at least to some degree, and often suffer dents from impacts which act as severe imperfections. Buckling of imperfect quasibrittle structures generally leads to snapthrough instability which typically exhibits size effect on the nominal strength.

Delamination in sandwiches and laminate composites has traditionally been analyzed by strength theory (either elasto-plasticity or elasticity with strength limit). This classical theory implies no size effect. However, according recent experiments by Bažant et al. [1] and Boyden et al. [2], the size effect in typical sandwich plates is transitional between the strength theory and linear elastic fracture mechanics. Therefore, the structure is quasibrittle, which means that the size of the fracture process zone (FPZ) cannot be considered to be negligible to the cross section dimension of normal-size sandwich structures. So, delamination fracture is modeled by a cohesive crack model, rather than LEFM or strength theory.

The present study relies on numerical simulations using geometrically nonlinear finite element analysis as well as the softening foundation model, which is an adaptation of Winkler elastic foundation. Dimensionless variables are used to cover the entire practical range. Both shortwave and longwave wrinkling are considered.

\section{Softening foundation model}

The analysis of delamination in sandwich structures subjected to pure bending, as shown in Figure 1a, can be simplified by modeling the skin as an axially compressed beam supported by a softening foundation consisting of independent continuously distributed nonlinear springs Figure $1 \mathrm{~b}$. For the mathematically analogous problem of a foundation with bilinear elastic-plastic hardening response, the solution is available [3]. Here the problem is solved for bilinear elastic-softening response, in which the softening represents gradual decohesion due to a cohesive crack under the beam. The differential equation of the problem reads

$$
E_{s} I_{s} \frac{d^{4} W}{d X^{4}}+P \frac{d^{2} W}{d X^{2}}+F=-P \frac{d^{2} W^{\circ}}{d X^{2}}
$$

where $E_{s}$ is the Young's modulus of the skin, $I_{s}=t^{3} / 12$ is the moment of inertia (per unit width) of the cross section of the skin of thickness $t, P$ is the axial force in the beam (per unit width), $X$ in the coordinate in the axial direction and $W(X)$ is the deflection (lateral displacement) of the skin, additional to the initial deflection $W^{\circ}$. 
(a)

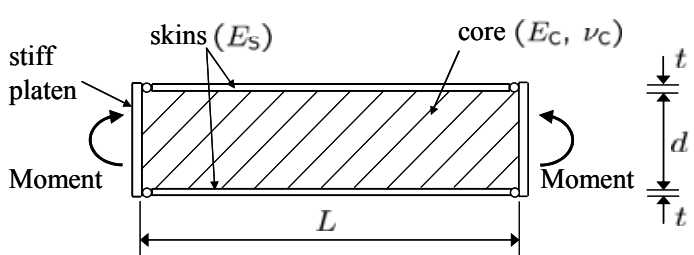

(b)

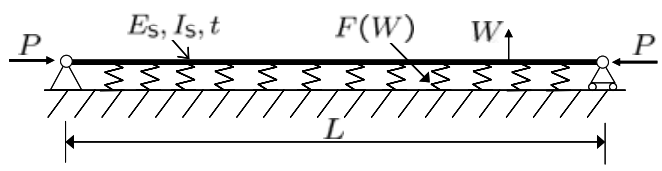

(c)

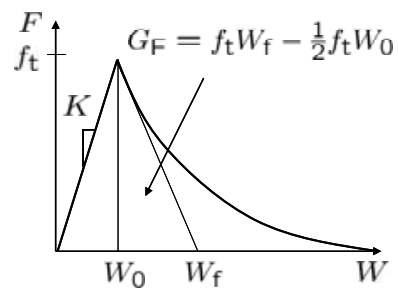

(d)

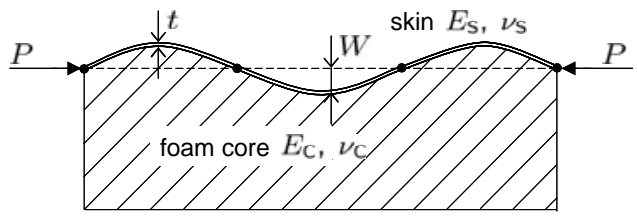

(e)

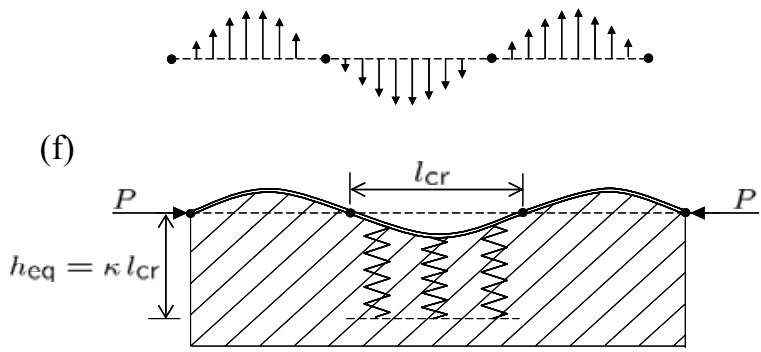

(g)

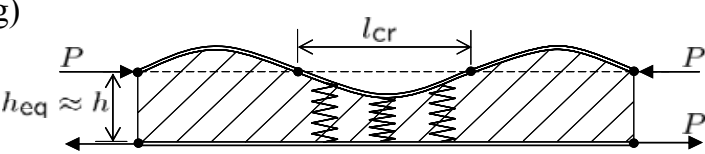

(h)

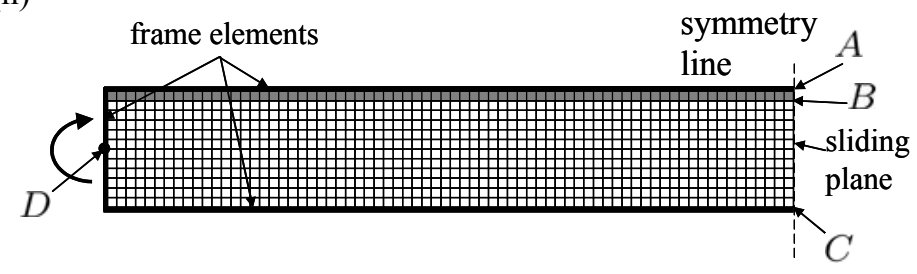

Figure 1: (a) The geometry of a typical sandwich beam subjected to pure bending. (b) The beam subjected to an axial compression force $P$ supported by a softening foundation. (c) The force-displacement relation of the softening foundation. (d) The deflection of the top skin. (e) Equilibrated stress acting on the foam. (f) Equivalent height for shortwave and (g) longwave wrinkling. (h) The finite element mesh.

Furthermore, $F$ is the distributed lateral force (traction), defined as

$$
F= \begin{cases}K W & \text { if } W \leq W_{0} \\ K W_{0} \mathrm{e}^{-\left(W-W_{0}\right) /\left(W_{f}-W_{0}\right)} & \text { if } W>W_{0}\end{cases}
$$

where $K$ is the foundation modulus (i.e., the spring stiffness of the foundation per unit length), $W_{0}$ is the displacement at which the tensile strength $f_{t}$ is reached (Figure 1c).

$$
G_{F}=f_{t}\left(W_{f}-W_{0} / 2\right)
$$

$G_{F}$ represents the area under the stress-displacement curve in Figure 1c. The distributed spring stiffness $K$ (per unit length of the beam) may be interpreted as

$$
K=E_{c} / h_{e q}
$$


Where $E_{c}$ is Young's modulus of the sandwich core, and $h_{e q}$ represents the equivalent (or effective) depth of the foundation.

For shortwave wrinkling the wave length $L_{c r}<<h \quad(\mathrm{~h}=$ core thickness). In that case the core may be regarded as an infinite half space. The reason is that the alternating tractions applied on the core by the periodically wrinkled skin (Figure 1e) are self-equilibrated over a segment of length $2 L_{c r}$ where $L_{c r}$ is the halfwavelength of the skin buckling (Figure 1f). Therefore, according to the Saint-Vernant principle, the stresses caused by periodic wrinkling must exponentially decay to nearly zero over a distant form the skin roughly equal to $2 L_{c r}$. Therefore, it must be possible to write

$$
h_{e q 0}=\kappa L_{c r}
$$

where $\kappa$ is some constant and subscript 0 refers to the limit case $L_{c r} / h \rightarrow 0$.

This leads to an expression for the critical axial compression force in the skin at bifurcation (see Bažant and Grassl [4] for details) as

$$
P_{c r 0}=2 \sqrt{K E_{s} I_{s}}=k_{1}\left(E_{c}^{\prime 2} E_{s}\right)^{1 / 3} t \quad \text { where } \quad k_{1}=\left(2 / 3 \kappa^{2} \pi^{2}\right)^{1 / 3}
$$

Note that this expression has the same form as derived in Hoff and Mautner [5], if $\kappa=\alpha \sqrt{1+v_{c}}$ with $\alpha=0.43$. Here, however, $\alpha=0.53$ is used, as determined from a single finite element analysis.

Consider now the case of longwave wrinkling $\left(L_{c r}>>h\right)$, shown in Figure 1g, and that the sandwich beam is subjected to bending moment only (i.e. with no axial force). Then the opposite skin is under tension and may be approximated as rigid base, with no deflection. The transverse compressive stress in the core is now almost uniform, and

$$
h_{e q \infty}=h
$$

i.e., the foundation stiffness $K=E_{c}^{\prime} / h_{e q}$ is constant (independent of the critical wavelength). The critical compressive force in the skin at bifurcation for periodic skin buckling (without delamination) is

$$
P_{c r o o}=2 \sqrt{K E_{s} I_{s}}=\sqrt{E_{c}^{\prime} E_{s} t^{3} / 3 h}
$$

where subscript $\infty$ refers to the limit case $L_{c r} / h \rightarrow \infty$, for which the solution is exact. The same expression was reported in Heath [6].

The transition between shortwave and longwave wrinkling is smoothly distributed over a certain dimensionless variable

$$
\varsigma=h_{e q 0} / h
$$

The shortwave bound $h_{e q}=h_{e q 0}$ must be tangentially approached for $\varsigma \rightarrow 0$, and the longwave bound $h_{e q}=h_{e q \infty}$ must be an asymptote for $\varsigma \rightarrow \infty$.

An expression that meets all the asymptotic conditions is

$$
h_{e q 0} / h_{e q}=\zeta+e^{-\left(\zeta+a_{1} \zeta^{2}+a_{2} \zeta^{3}\right)}
$$

where $a_{1}=0.24$ and $a_{2}=0.36$, as obtained by fitting to linear elastic finite element results with the MarquardtLevenberg algorithm for nonlinear least-squares optimization.

\section{Formulation in Dimensionless Variables}

The solution may generally be expressed as a relation among seven dimensional variables: $E_{s} I_{s}, K, P, W_{0}, W^{0}, W_{f}, x$ which involve 2 independent dimensions, force and length. According to Vashy-

Buckingham theorem of dimensional analysis, the number of dimensionless variables governing the problem is 7 $-2=5$. They may be chosen the same as in a previous study of plastic bilinearly hardening foundation [3]:

$$
x=X\left(E_{s} I_{s} / K\right)^{-1 / 4}, \lambda=\frac{1}{2} P\left(K E_{s} I_{s}\right)^{-1 / 2}, w=W / W_{0}, w^{\circ}=W^{\circ} / W_{0}, w_{f}=W_{f} / W_{0}
$$

Substituting these equations and Eq. (2) in Eq. (1), yields the dimensionless differential equation: 


$$
\begin{gathered}
\frac{d^{4} w}{d x^{4}}+2 \lambda \frac{d^{2} w}{d x^{2}}+w=-2 \lambda \frac{d^{2} w^{\circ}}{d x^{2}} \text { if } w \leq 1 \\
\frac{d^{4} w}{d x^{4}}+2 \lambda \frac{d^{2} w}{d x^{2}}+e^{(w-1) /\left(w_{f}-1\right)}=-2 \lambda \frac{d^{2} w^{\circ}}{d x^{2}} \text { if } w>1
\end{gathered}
$$

where $w$ is the dimensionless deflection. For a perfect beam $\left(w^{\circ}=0\right)$, the first eigenmode of buckling at bifurcation is determined from Eq. (12) as $w=\sin x$, and the corresponding load at bifurcation results in $\lambda=1$. A general imperfection of skin may be expressed as a combination of all eigenmodes of buckling. The first eigenmode may be expected to have dominant influence. Therefore, the imperfection $\delta$ of the skin is chosen to be proportional to the aforementioned displacement profile $w=\sin x$ of a perfect skin at first bifurcation, i.e. $w^{\circ}=\delta \sin x$. The solution of () for the elastic case $\left(w_{\max }<1\right)$, with the aforementioned imperfection, is

$$
w(x)=\frac{\lambda \delta}{1-\lambda} \sin x
$$

The solution will be used for deriving the size effect law.

The dimensionless variables $x, w, w^{\circ}$ and $\lambda$ are size independent. However, ensuring constant fracture energy (in the sense of the crack band model) requires that the dimensionless parameter $w_{f}$ be considered size dependent, as obtained by inserting (3) into (11);

$$
w_{f}=\frac{G_{F} E_{c}}{f_{t}^{2} h_{e q}}+\frac{1}{2}
$$

Parameters $G_{F}, E_{c}$ and $f_{t}$ are material properties independent of the structure size, while $h_{e q}$ is proportional to the structure size. Thus, the size dependence of $w_{f}$ can be characterized as

$$
w_{f}=\frac{1}{\xi}+\frac{1}{2}
$$

where

$$
\xi=h_{e q} / l_{0}, \quad l_{0}=E_{c} G_{F} / f_{t}^{2}
$$

$\xi$ is dimensionless and $l_{0}$ is known as Irwin's characteristic material length, approximately characterizing the fracture process zone length. To simplify analysis, only one half of the beam is modeled and symmetric deformation is assumed.

\section{Geometrically nonlinear finite element analysis}

To determine parameter $\alpha$ and to validate the simplified modeling of delamination by the softening foundation model, a geometrically nonlinear finite element program (FEAP) is used. A sandwich beam, depicted in Figure 1a, is considered and is modeled using the finite element mesh in Figure 1h. The skins are represented by beam elements taking into account large displacements and large rotations. For the core, plane stress finite elements based on a linearized small displacement formulation is used. The core is treated as isotropic, and for the skin only the longitudinal elastic modulus $E_{s}$ needs to be considered since the transverse and shear moduli of laminate skin are immaterial for bending and axial deformation.

The beam is considered to be subjected to a uniform bending moment, $M$. However, as long as the core thickness $h$ is large enough for the stresses from wrinkling to decay to nearly zero over the core thickness, the only loading that matters is the axial force. Whether this force is produced by moment alone, or a combination of bending moment and axial fore, is immaterial.

An elastic stress-strain relation is used for all the elements of the core except a narrow band of elements under the skin (marked gray in Figure 1h). It is known that the delamination fracture occurs within the core very near the interface with the skin, but not within the interface. Therefore, perfect bond between the skins and the core is enforced, Transverse softening of the aforementioned band, which can be regarded as distributed microcracking, simulates delamination. In the softening band, the stress-strain law is elastic in the pre-peak, and the post-peak response follows the isotropic damage model, which is defined as

$$
\sigma=(1-\omega) D_{c}: \varepsilon
$$


Here $\sigma$ and $\varepsilon$ are the stress and strain tensors in the core, $\omega$ is the damage variable, and $D_{c}$ is the isotropic elastic stiffness tensor of the core, which is based on the Young's modulus $E_{c}$ and the Poisson's ratio $v_{c}$. The damage law is chosen so that it results in an exponential stress-strain curve in uniaxial tension. The inelastic strains determined by the isotropic damage model are fully reversible, i.e. the secant stiffness points toward the origin (this reversibility would, of course, be unrealistic if crack unloading were not nonexistent in the present simulations).

As before, only half of the beam is modeled. The loading moment $M$ is applied at point $D$ (Figure $1 \mathrm{~h}$ ) and is assumed to be transferred by a rigid loading platen into the upper and lower skins. The structure is restrained in longitudinal direction at point $\mathrm{A}$. The loading is controlled by prescribing the displacement of point $B$. The same initial displacement, i.e. $w^{\circ}=\delta \sin x$, is prescribed for the upper skin. The imperfection amplitude at the middle of the beam at point $A$ is slightly increased in the same way as for the softening foundation model, to control the place where the delamination begins.

\section{Results and comparison of softening foundation to finite elements}

The effect of the structure size on the relation between the load parameter $\lambda$ and the mid-point displacement $w_{a}=w(L / 2)$ is shown, for three imperfection amplitudes $\delta=0.1,1,2$, in Figure 2a-c. As one can see, the results of the softening foundation model are in reasonable approximate agreement with the more accurate finite element results. The comparison shows that the size has a strong effect on the post-peak part of the load-displacement relation. The larger the size, the less energy is dissipated in relation to the energy dissipated by delaminating the entire skin. Furthermore, a closer examination of the size effect on the evolution of the diagram of load versus blister length (which is the length in the middle potion of the beam in which $w>1$ ) reveals a size effect on the nominal strength, see Figure $2 d-f$. The larger the size, the smaller is $\lambda_{\max }$. Furthermore, note that the size effect intensity depends strongly on the imperfection amplitude. A law for this size effect is proposed next.

\section{Size effect law for imperfection sensitive wrinkling}

The size effect on the dimensionless nominal strength, $\lambda_{N}=\lambda_{\max }$, shown in Figure $2 \mathrm{~g}$, has a form similar to the size effect law for crack initiation in quasibrittle structures proposed by Bažant [7,8]. This law, however, is not directly applicable since imperfections are seen in Figure $2 \mathrm{~g}$ to influence the size effect. Therefore, a generalized law of the form

$$
\lambda_{N}(\delta, \xi)=\lambda_{\infty}(\delta)\left[1+\frac{1}{k(\delta)+a \xi^{b}}\right], \quad k(\delta)=c \delta^{-d}
$$

is proposed here, with constants $a, b, c, d$ and parameters $\lambda_{\infty}$ and $k$ depending on the imperfection amplitude $\delta$. For large sizes $(\xi \rightarrow \infty)$, the nominal strength is decided by crack initiation $(w=1)$, and in that case Eq. (19) leads to

$$
\lambda_{N}(\delta, \infty)=\lambda_{\infty}=1 /(1+\delta)
$$

For small sizes $(\xi \rightarrow 0)$, the nominal strength in (19) turns into

$$
\lambda_{N}(\delta, 0)=\lambda_{\infty}(\delta)\left[1+\frac{1}{k(\delta)}\right]
$$

Parameters $a, b, c, d$ in Eq. (19) are determined as optimal fits of numerical results using the Marquardt-Levenberg algorithm for nonlinear least-squares optimization. First the parameters $c$ and $d$ are determined from the fit of the results for the smallest size $(\xi=0.001)$ in Figure $2 \mathrm{~g}$, for varying imperfections. Then the parameters $a$ and $b$ in Eq. (19) are fitted fore the largest imperfection $(\delta=6)$ and varying size. The optimum values are $a=9.94$, $b=1.2, c=6.82, d=1.21$. The size effect law in Eq. (19) using these parameters is compared to the results of the softening foundation model in Figure $2 \mathrm{~g}$. The approximation is seen to be satisfactory. 
(a)

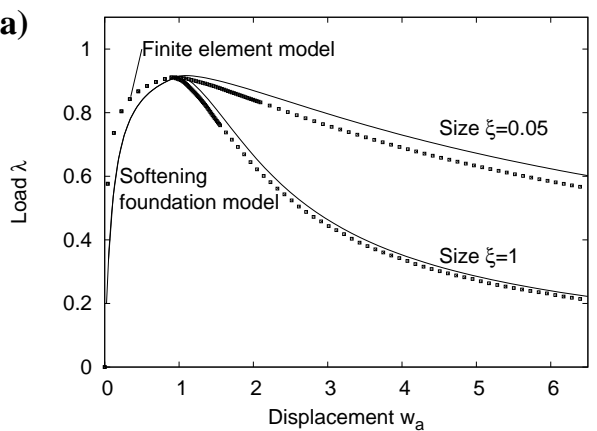

(b)

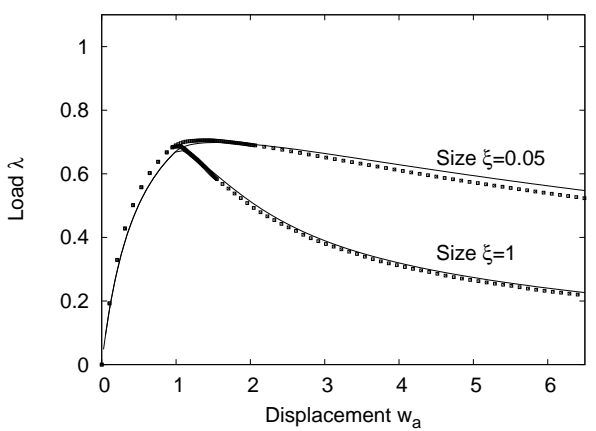

(c)

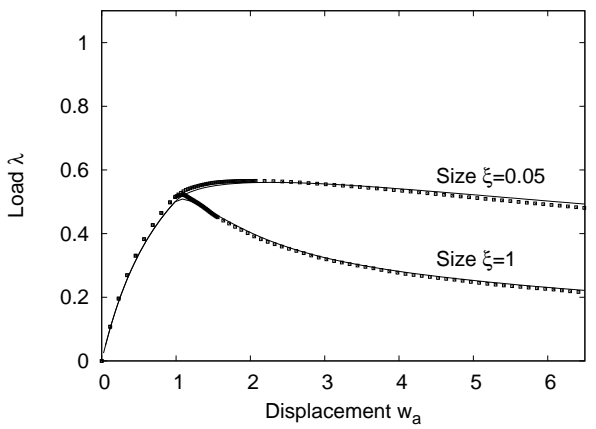

(d)

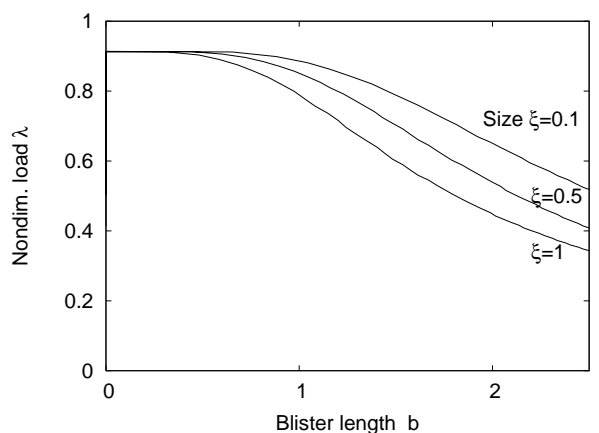

(e)

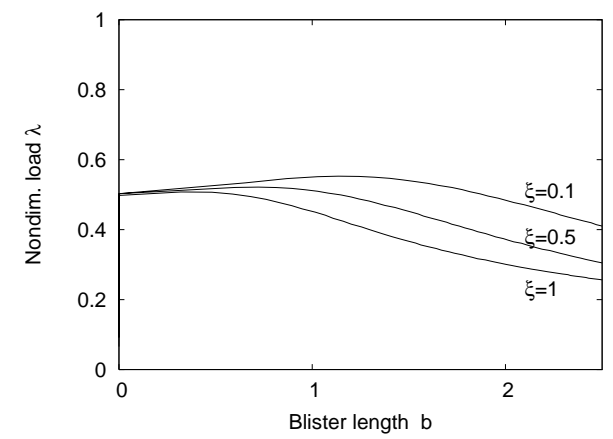

(f)

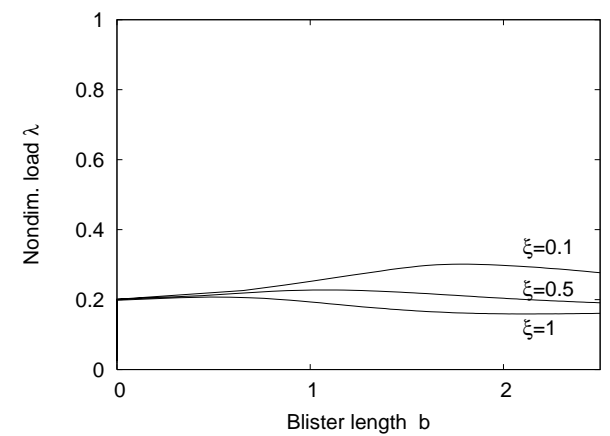

(g)

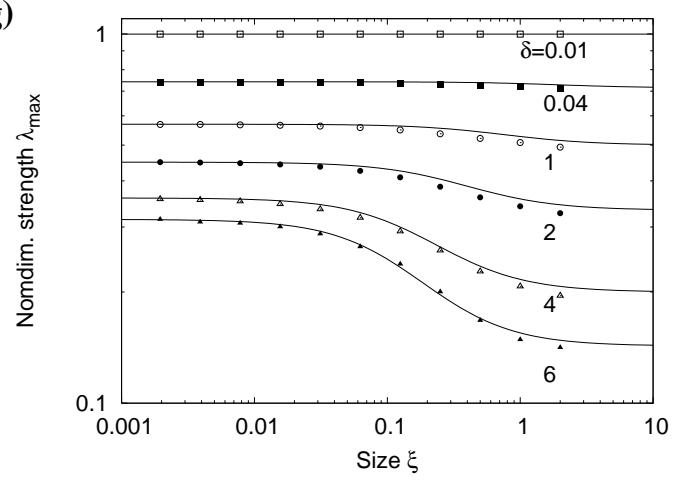

Figure 2: (a)-(c): The load $\lambda$ versus the mid-point displacement $w_{a}$ obtained with the softening foundation model and the finite element model for the imperfections (a) $\delta=0.1$, (b) $\delta=0.5$, (c) $\delta=1$ for two sizes $(\xi=1$ and $\xi=$ 0.05 ). (d)-(f): The load $\lambda$ versus the blister length (mid beam region in which $w>1$ ) of the upper skin for the imperfections (d) $\delta=0.1$, (e) $\delta=1$, (f) $\delta=4$. (g) Comparison of the size effect law with the nominal strength-size relations obtained with the softening foundation model for different imperfections. 


\section{Conclusions}

1. In view of recent experiments revealing a size effect transitional between LEFM and strength theory, the delamination fracture of laminate-foam sandwich structures must be treated as a cohesive crack with a softening stress-separation relation characterized by both fracture energy and tensile strength. In contrast to LEFM, no preexisting interface flaw needs to be considered.

2. The skin (or facesheet) can be treated as a beam on elastic foundation, provided that the equivalent (or effective) core depth $h_{e q}$ for which the hypothesis of uniform transverse stress gives the correct foundation stiffness is considered to depend on the critical wavelength $L_{c r}$ of skin wrinkles; $h_{e q}=$ core thickness $\mathrm{h}$ for the asymptotic case of longwave wrinkling ( $L_{c r} / h \rightarrow \infty$ ), while (because of St. Venant principle) $h_{e q}$ is proportional to $L_{c r}$ for the asymptotic case of shortwave wrinkling $\left(L_{c r} / h \rightarrow 0\right)$.

3. A properly formulated softening foundation model can give good agreement with geometrically nonlinear finite element analysis of delamination fracture triggered by wrinkling.

4. Although the nominal strength of sandwich structures failing by wrinkling-induced delamination fracture is size independent when there is no imperfection, it becomes strongly size dependent with increasing imperfection.

5. Introduction of proper dimensionless variables makes it possible to cover with numerical simulations the entire practical range, and fitting the dimensionless numerical results for cohesive delamination fracture with a formula for correct shortwave and longwave asymptotics allows constructing an approximate size effect law for nominal strength of arbitrarily imperfect sandwich beams subjected to uniform bending moment.

6. There is also a strong size on postpeak energy absorption by a sandwich structure, both in presence and absence of imperfections. This is important for impact and blast resistance.

\section{Further Accomplishments}

a) Nanoscale-Based Mechanical-Statistical Assessment of Quasibrittle Failure Risk and Necessity of Size Effect on Safety Factors. In the design of ships as well as other structures, one must ensure an extremely low failure probability such as $10^{-6}$. How to do that has been adequately understood only for the limiting cases of brittle or ductile structures. Developed has been a theory to do that for the transitional class of quasibrittle structures, having brittle constituents and characterized by nonnegligible size of material inhomogeneities. We show that the probability distribution of strength of the representative volume element (RVE) of material is governed by the Maxwell-Boltzmann distribution of atomic energies and the stress dependence of activation energy barriers. This distribution is statistically modelled by a hierarchy of series and parallel couplings. It consists of a broad Gaussian core having a grafted far-out power-law tail with zero threshold and amplitude depending on temperature and load duration. With increasing structure size, the Gaussian core shrinks and Weibull tail expands according to the weakest-link model for a finite chain of RVEs. The model captures experimentally observed deviations of the strength distribution from Weibull distribution, and of the mean strength scaling law from a power law. These deviations can be exploited for verification and calibration. The proposed theory will increase the safety of composite parts ships (as well as aircraft) and allow designs closer to the safety margin.

b) Postcritical Imperfection Sensitivity of Sandwich Columns Soft in Shear and in Transverse Deformation. Buckling of all columns, including sandwich columns, has generally been considered to be imperfection insensitive. The latest investigation, however, has shown, surprisingly, that this is not true for navy-type sandwich plates. This investigation extends to the initial postcritical behavior the previous year study that led to the energetic variational solution of critical loads and of the choice of finite strain measure for structures very weak in shear, remaining in a state of small strain. For the postcritical behavior, consideration of the transverse deformation is found to be essential. It transpires that imperfection sensitivity of such structures, particularly laminate-foam sandwich plates, can arise for a certain range of stiffness and geometric parameters, depending on the proper value of parameter $m$ of the Doyle-Ericksen finite strain tensor, as determined in the previous analysis. The bifurcation is symmetric and Koiter's 2/3-power law is found to be followed. The analytical predictions of maximum load reductions due to imperfection sensitivity are verified by finite element simulations. The possibility of interaction between different buckling modes, particularly lateral deflection and bulging, is also detected, with the conclusion that lateral deflection dominates in common practical situations. 
Acknowledgment: Supported by ONR Grant N00014-02-I-0622, Boeing Co. Grant, and Federal Aviation Administration Grant 01-C-AW-NWU, all to Northwestern University.

\section{References}

1. Z. P. Bažant, Y. Zhou and I. M. Daniel. Size effect on strength of laminate-foam sandwich plates. Journals of Engineering Materials and Technology. 2006. In press.

2. J. Bayldon, Z. P. Bažant, I. M. Daniel and Q. Yu. Size effect on compressive strength of laminate-foam sandwich plates. Journal of Engineering Materials and Technology. 2006 . In press.

3. V. Tvergaard and A. Needleman. On the localization of buckling patterns. Journal of Applied Mechanics, ASME, 47:613619, 1980.

4. Z. P. Bažant and P. Grassl. Size effect of cohesive delamination fracture triggered by sandwich skin wrinkling. 2006. AMSE J. of Appl. Mech., submitted to.

5. N. J. Hoff and S. E. Mautner. The buckling of sandwich-type panels. Journal of Aeronautical Sciences, 12:285-297, 1945.

6. W. G. Heath. Sandwich construction, part 2: The optimum design of flat sandwich panels. Aircraft Engineering, 32:230$235,1960$.

7. Z. P. Bažant. Scaling of quaasibrittle fracture: I. Asymptotic analysis based on laws of thermodynamics. II. The fractal hypothesis, its critique and Weibull connection. Report no. 95-7/c402s, Dep. Of Civil Engineering, Northwestern University, Evanston, Illinois 60209, U.S.A, 1995.

8. Z. P. Bažant. Scaling of quasi-brittle fracture: asymptotic analysis. International Journal of Fracture, 83:19-40, 1997.

\section{Further Publications on This Grant by Principal Investigator:}

Bazant, Z.P., (2005). "Scaling of structural strength", $2^{\text {nd }}$ ed. (paperback), Dover, New York.

Bazant, Z.P., Zhou, Y., Daniel, I.M., Caner, F.C., and Yu, Q. (2006). "Size Effect on Strength of Laminate-Foam Sandwich

Plates", ASME J. of Materials and Technology, in press.

Cervenka, J., Bazant, Z.P., and Wierer, M. (2005). "Equivalent localization element for crack band approach to meshsensitivity in microplane model.” Int. J. for Numerical Methods in Engrg. 62 (5), 700-726.

Bazant, Z.P., and Beghini, A. (2005). "Which formulation allows using a constant shear modulus for small strain-buckling of soft-core sandwich structures?” J. of Applied Mechanics ASME 72 (Sept.), 785-787.

Di Luzio, G., and Bazant, Z.P. (2005). "Spectral analysis of localization in nonlocal and over-nonlocal materials with softening plasticity or damage." Int. J. of Solids and Structures 42, 6071-6100.

Bazant, Z.P., Guo, Z., Espinosa, H., Zhu, Y. and Peng, B. (2005). "Epitaxially influenced boundary layer model for size effect in thin metallic films." J. of Applied Physics 97, 073506-1 - 073506-13.

Bazant, Z.P., and Beghini, A. (2006). "Stability and finite strain of homogenized structures soft in shear: sandwich or fiber composites, and layered bodies." Int. J. of Solids and Structures 43, 1571-1593.

Bazant, Z.P., and Pang, S.-D. (2006). "Mechanics based statistics of failure risk of quasibrittle structures and size effect on safety factors." Proc. of the National Academy of Sciences 103, in press.

Bazant, Z.P., and Pang, S.-D. (2006). "Activation energy based extreme value statistics and size effect in brittle and quasibrittle fracture". J. of the Mechanics and Physics of Solids 54, in press.

Cusatis, G., Bazant, Z.P., and Cedolin, L. (2006). "Confinement-shear lattice CSL model for fracture propagation in concrete." Computer Methods in Applied Mechanics and Engrg., in press.

Bazant, Z.P., Zhou, Y., and Daniel, I.M. (2006). "Size effect on strength of laminate-foam sandwich plates", J. of Engrg. Materials and Technology ASME 128, in press.

Bayldon, J., Bazant, Z.P., Daniel. I.M., and Yu, Q. (2006). "Size effect on Compressive Strength of Laminate-Foam Sandwich Plates." J. of Engrg. Materials and Technology ASME 128, in press.

Bazant, Z.P., Vorechovsky, M., and Novak, D. (2006). "Asymptotic prediction of energetic-statistical size effect from deterministic finite element solutions.” J. of Engrg. Mech. ASCE 128, in press.

Beghini, A., Bazant, Z.P., Waas, A.M., and Basu, S. (2006). "Postcritical Imperfection Sensitivity of Sandwich Columns Soft in Shear and in Transverse Deformation", Int. J. of Solids and Structures, submitted to.

Bazant, Z.P., and Pang, S.D. (2006). "Activation energy based extreme value statistics and size effect in brittle and quasibrittle fracture." J. of the Mechanics and Physics of Solids, submitted for publ.

Vorechovsky, M., Bazant, Z.P., and Novak, D. (2005). "Procedure of statistical size effect prediction for crack initiation problems.” Proc. (CD) 11-th Int. Conf. on Fracture (ICF-11, held in Turin,

Italy), A. Carpinteri, ed., Paper 40-6-1, pp. 1-6.

Bazant, Z.P., and Pang, S.-D. (2005). "Revision of Reliability Concepts for Quasibrittle Structures and Size Effect on Probability Distribution of Structural Strength.” Safety and Reliability of Engrg. Systems and Structures (CD) (Proc., 8th Int. Conf. on Structural Safety and Reliability, ICOSSAR 2005, held in Rome), G. Augusti, G.I. Schueller and M. Ciampoli, eds., Millpress, Rotterdam, pp. 377-386.

Bazant, Z.P., Vorechovsky, M., and Novak, D. (2005). "Role of deterministic and statistical length scales in size effect for quasibrittle failure at crack initiation." ibid., pp. 411-415. 